\section{Myelodysplastische Syndrome}

\section{Welche Patienten brauchen Deferasirox?}

Zwei von drei Patienten mit myelodysplastischen Syndromen (MDS) benötigen dauerhaft Bluttransfusionen. Dies kann bei MDS-Patienten bereits nach kurzer Zeit zu einer chronischen Eisenintoxikation führen und erfordert daher eine Chelat-Therapie. Exjade ${ }^{\circledR}$ (Deferasirox) stellt eine wirksame, gut verträgliche und einmal täglich oral einzunehmende Alternative zu den bisher verfügbaren Chelatoren dar.

MDS sind eine heterogene Gruppe von klonalen Knochenmark-Erkrankungen, bei denen die Hämatopoese einer oder mehrerer Zelllinien unzureichend ist. Rund ein Drittel aller MDS-Erkrankungen mündet in eine akute myeloische Leukämie (AML). Etwa zwei Drittel aller MDS-Patienten benötigen eine dauerhafte Transfusionstherapie. Da MDS überwiegend bei über 60-Jährigen auftreten, ist im Zuge der gegenwärtigen demografischen Entwicklung in den nächsten Jahren mit einer erheblichen $\mathrm{Zu}$ nahme der Erkrankung zu rechnen. Zurzeit beträgt die jährliche Inzidenz der MDS 5:100.000, bei über 70-Jährigen 40:100.000.

\section{Deferasirox ist ein Durchbruch in der oralen Eisenchelation}

Eine längerfristige Transfusionstherapie kann zu einer Eisenüberladung des Körpers führen. Das nicht transferringebundene und freie Eisen jedoch fördert die Bildung hochreaktiver Hydroxylradikale, die wiederum toxisch wirken. Aus der Eisenintoxikation können Funktionsstörungen von Herz, Leber und endokrinen Organen resultieren. Einer solchen transfusionsbedingten Eisenintoxikation kann wirksam mit Chelatoren begegnet werden: sie binden freies Eisen und bilden einen Chelatkomplex, der ausgeschieden werden kann. Die Standardbehandlung bestand bisher aus der Gabe langsamer s.c. Infusionen des Chelatbildners Deferoxamin über 8-12 h an 5-7 Wo- chentagen. Seit August 2006 ist der Chelatbildner Deferasirox unter dem Warenzeichen Exjade ${ }^{\circledR}$ europaweit zugelassen. Er ist der einzige Eisenchelator, der einmal täglich oral eingenommen wird und kontinuierlich über $24 \mathrm{~h}$ wirkt. Der Einnahmemodus stellt gegenüber der parenteralen Behandlung eine erhebliche Erleichterung für die Patienten dar. Lebensbedrohliche Agranulozytosen wie unter dem oralen Chelator Deferipron wurden unter Deferasirox bisher nicht beschrieben. Prof. Norbert Gattermann, Düsseldorf, bezeichnet die Einführung von Deferasirox daher als «Durchbruch in der Behandlung der transfusionsbedingten Eisenüberladung». Wegen einzelner Fälle von akutem Nierenversagen und Leberfunktionsstörungen sollten Leber- und Nierenfunktionsparameter regelmäßig beobachtet werden. Bei unklarer Zytopenie unter Deferasirox sei die Unterbrechung der Behandlung in Erwägung zu ziehen [1]. Zu einem späteren Zeitpunkt kann die Behandlung in Abhängigkeit von den individuellen klinischen Umständen wieder begonnen werden.

\section{Gute Prognose rechtfertigt den Ein- satz einer Eisenchelat-Therapie}

Die Chelat-Therapie der Eisenintoxikation ist für die Thalassämia major am besten untersucht. Bei MDS muss der Anteil der Eisenintoxikation an der Sterblichkeitsrate von Patienten Gattermann zufolge noch genauer untersucht werden. Eine
Studie, die auf dem ASH-Kongress 2006 vorgestellt wurde, deutet aber auf eine wesentliche Verbesserung des Langzeitüberlebens durch eine Eiseneliminationstherapie hin [2]. Für eine Eisenchelation kommen vor allem Patienten mit einer relativ guten Prognose und daher mit der Aussicht auf eine lang anhaltende Transfusionsbehandlung in Frage. In einer retrospektiven Auswertung von 467 Männern und Frauen im Alter von 22-93 Jahren mit de novo diagnostiziertem MDS stellten Malcovati et al. [3] einen klaren Zusammenhang zwischen MDS-Typ und Überlebensprognose fest (Abb. 1). Der «Konsensus von Nagasaki» aus dem Jahr 2005 empfiehlt die Therapie der Eisenüberladung unter anderen bei folgenden MDS-Patienten:

- Patienten mit regelmäßigem Transfusionsbedarf - MDS mit niedrigem Risiko

- MDS-Typen RA, RARS und 5q nach WHO

- Kandidaten für eine allogene Stammzelltransplantation

- Längerfristig stabiler Krankheitsverlauf

- Serumferritinwerte $>1000-2000 \mathrm{ng} / \mathrm{ml}$ oder sonstiger Nachweis einer chronischen Eisenüberladung

- Keine schwere Beeinträchtigung der Prognose durch Begleiterkrankungen

Bei MDS-Patienten, die eine allogene Stammzelltransplantation erhalten, erhöht eine Eisenintoxikation vor der Transplantation das Ereignisund Sterberisiko [4]. Daher sind auch diese Patienten Kandidaten für eine Eisenchelation vor dem Eingriff. Zur prognostischen Einschätzung des Risikos bei MDS-Patienten wird das International Prognostic Scoring System (IPSS) empfohlen (Tab. 1). Es definiert die entscheidende prognostische Bedeutung der Karyotypveränderungen und lässt damit Risikogruppen diskriminieren, die durch die WHO-Klassifikation allein nicht hinreichend erfasst sind. Es ist mittlerweile breiter Konsens, dass MDS-Patienten in den beiden niedrigsten Risikostufen des IPSS (low, int-1) einen Nutzen von der Chelat-Therapie erwarten können.

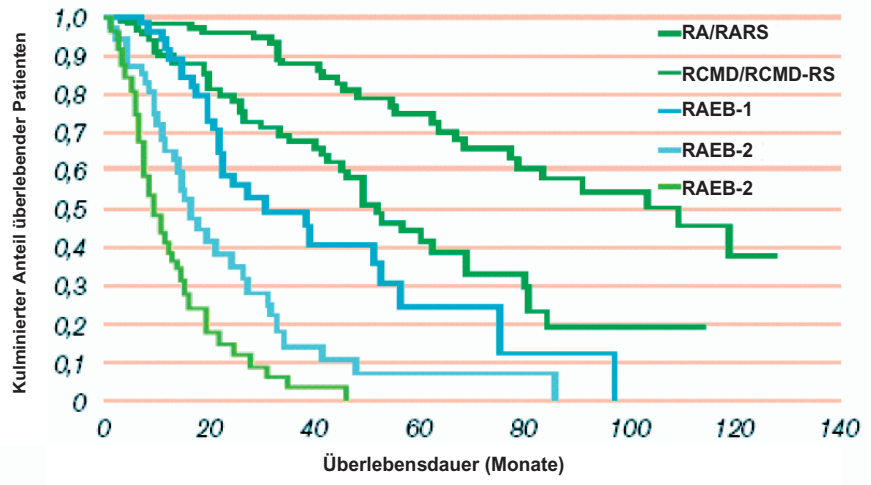

Abb. 1. Kumulatives Überleben nach WHO (nach [3])

\begin{tabular}{llllll}
\hline Prognosefaktor & 0 & 0,5 & 1 & 1,5 & 2 \\
\hline Blastenanteil im & $<5$ & $5-10$ & - & $11-20$ & $21-30$ \\
Knochenmark, $\%$ & & & & \\
Karyotyp* & günstig & intermediär & ungünstig & - & - \\
Anzahl der betroffenen & $0-1$ & $2-3$ & - & - & - \\
Zellreihen & & & & \\
\hline *Karyotypanomalien mit Niedrigrisiko: normal, , del(5q), del(20q), -Y. & \\
Karyotypanomalien mit intermediären Veränderungen: alle übrigen Anomalien. & \\
Karyotypanomalien mit Hochrisiko: komplex, Monosomie 7. & \\
\hline Risikogruppe & Score-Summe & Mittlere Überlebenszeit (Median), Jahre \\
\hline Niedrigrisiko & 0 & 5,7 & \\
Intermediär 1 (int-1) & $0,5-1$ & 3,5 & \\
Intermediär 2 (int-2) & $1,5-2$ & 1,2 & \\
Hochrisiko & $\geq 2,5$ & 0,4 & \\
\hline
\end{tabular}

Tab. 1. International Prognostic Scoring System (IPSS) für MDS (nach [5]) 
Kasuistik

\section{Einsatz der Eisenchelat-Therapie bei Patienten mit myelodysplastischen Syndromen (MDS)}

Regelmäßige Bluttransfusionen können bereits nach kurzer Zeit zu einer Eisenintoxikation führen, da der menschliche Körper nur eine geringe physiologische Ausscheidungsaktivität für Eisen besitzt. Bei Patienten mit transfusionspflichtigen MDS und guter Prognose ist deshalb der frühzeitige Einsatz einer Eisenchelat-Therapie empfehlenswert. Kandidaten für eine Stammzelltransplantation, die normale Serumferritinspiegel haben, weisen ein verbessertes Gesamtüberleben auf [4]. In diesem Zusammenhang steht auch folgende Kasuistik, die uns freundlicherweise von PD Dr. Uwe Platzbecker, Dresden, zur Verfügung gestellt wurde.

Ein 62-jähriger Patient stellt sich im April 2004 wegen zunehmender Müdigkeit und regelmäßig auftretendem Schwächegefühl vor, bei körperlicher Belastung komme er in letzter Zeit sehr schnell außer Atem. Der Patient befindet sich zu diesem Zeitpunkt in gutem Allgemeinzustand. Inspektion und körperliche Untersuchung weisen auf eine Anämie hin. Aufgrund der hämatologischen Laborbefunde wird ein MDS festgestellt und nach WHO als refraktäre Zytopenie mit Multilinien-Dysplasie (RCMD) klassifiziert. Die zytogenetische Untersuchung ist unauffällig, der IPSS-Score (International Prognostic Scoring System, s. Tab. 1) liegt bei 0 . Eine Indikation für Bluttransfusionen liegt zu diesem Zeitpunkt noch nicht vor.

\section{Chelat-Therapie wegen guter Pro- gnose}

Wegen zunehmender Anämie erhält der Patient ab Oktober 2004 eine Transfusionstherapie mit zwei Erythrozytenkonzentraten (EK) zunächst alle 4-6 Wochen, ab Dezember 2004 dann alle 4 Wochen. Dadurch kommt

Abb. 2. Entwicklung der Serumferritinwerte bei konstanter Dosierung von Deferasirox mit $20 \mathrm{mg} / \mathrm{kg} \mathrm{KG/Tag}$ (nach [6])

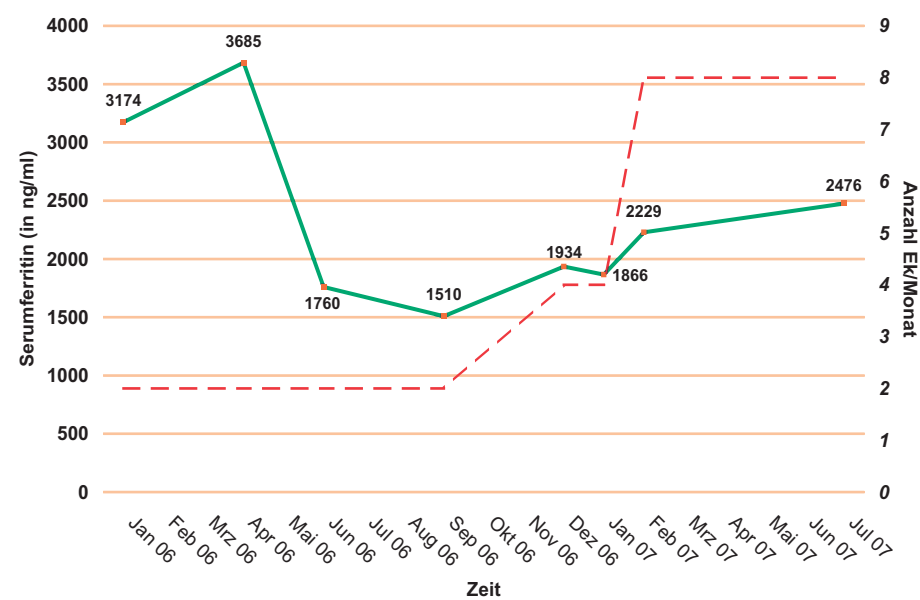

Stammzelltransplantation bei moderaten Ferritinwerten

Ab Dezember 2006 muss die Transfusionsfrequenz auf zwei EK alle 2 Wochen erhöht werden. Im Januar 2007 tritt ein Progress des MDS mit Übergang in eine chronische myelomonozytäre Leukämie, CMML-1 nach WHO, ein. In den peripheren Blutlymphozyten finden sich 5\% Blasten, zytogenetisch ein Karyotyp 46 XY, del. $5 q(31)$. Die fortschreitende Anämie macht zwei EK pro Woche erforderlich, worauf das Serumferritin bei gleichbleibender Deferasirox-Dosis von $20 \mathrm{mg} / \mathrm{kg} \mathrm{KG/Tag} \mathrm{im} \mathrm{Februar} 2007$ wieder über die 2000-ng/ml-Marke steigt.

Bis März 2007 nimmt der Blastenanteil zu, und die MDS geht in eine CMML-2 nach WHO über. Im April 2007 wird nach Identifikation eines HLA-identischen Spenders eine periphere Blutstammzelltransplantation durchgeführt, die erfolgreich verläuft: Das Blutbild normalisiert sich, Bluttransfusionen sind nicht mehr nötig. Bei weiterhin erhöhten Ferritinwerten ist in der Folgezeit eine kurzfristige Aderlassbehandlung geplant, die Therapie mit Deferasirox wurde deswegen beendet. Rückblickend waren der rechtzeitige Beginn und die konsequente Fortführung der Eisenchelat-Therapie möglicherweise wichtige Voraussetzungen für den günstigen Verlauf nach Transplantation.

Die Therapie der chronischen Eisenüberladung mit einem Eisenchelator ist wichtiger Baustein im therapeutischen Armentarium bei MDS-Patienten mit guter Prognose. Insbesondere Kandidaten für eine allogene Stammzelltransplantation profitieren möglicherweise besonders vom frühzeitigen Einsatz.

\section{Referenzen}

1 Gattermann N, Musch A: Arzneimitteltherapie 2007:25:240-247. 2 Leitch HA, et al: ASH 2006, \#249

3 Malcovati L et al.: J Clin Oncol 2005;23:1-10.

4 Armand P, et al.: Blood 2007;109:4586-4588.

5 Greenberg P, et al.: Blood 1997; 2079-2088.

6 Platzbecker U, Blutuntersuchung des Patienten, unveröffentlicht.

\section{Verfasser}

PD Dr. U. Platzbecker, Dresden

Dr. Thomas Bißwanger-Heim, Freiburg i. Br.

Impressum

Myelodysplastische Syndrome

Welche Patienten brauchen Deferasirox?

Kasuistik

Einsatz der Eisenchelat-Therapie bei Patienten mit myelodysplastischen Syndromen (MDS)

PharmaForum in ONKOLOGIE 30 | 8-9 | 07

C 2007 by S. Karger Verlag für Medizin und Naturwissenschaften $\mathrm{GmbH}, 79115$ Freiburg, Deutschland

Mit freundlicher Unterstützung von Novartis Oncology. 